\title{
Combination of immunotherapy with chemotherapy in first line treatment of metastatic gastric cancer? Too much, too little or just right?
}

\author{
Matthew C. H. $\mathrm{Ng}^{1}$, Su Pin $\mathrm{Choo}^{2}$ \\ ${ }^{1}$ Division of Medical Oncology, National Cancer Centre Singapore, Singapore, Singapore; ${ }^{2}$ Curie Oncology, Singapore, Singapore \\ Correspondence to: Su Pin Choo. Medical Oncologist, Curie Oncology, Singapore, Singapore. Email: choosupin@gmail.com. \\ Comment on: Kawazoe A, Yamaguchi K, Yasui H, et al. Safety and efficacy of pembrolizumab in combination with S-1 plus oxaliplatin as a first-line \\ treatment in patients with advanced gastric/gastroesophageal junction cancer: Cohort 1 data from the KEYNOTE-659 phase IIb study. Eur J Cancer \\ 2020;129:97-106.
}

Submitted Jun 14, 2020. Accepted for publication Sep 03, 2020.

doi: 10.21037/atm-20-4699

View this article at: http://dx.doi.org/10.21037/atm-20-4699

The benefit of immune check-point inhibitors (ICI) in patients with advanced gastroesophageal adenocarcinoma (GEA) has been demonstrated in the ATTRACTION-2 study $(1,2)$. In East Asian patients who failed two or more lines of systemic therapy, treatment with nivolumab monotherapy significantly improved overall survival (OS) compared to best supportive care. Tumour responses were very durable and treatment well tolerated. This benefit was independent of tumour PD-L1 score (TPS) leading to regulatory approval of nivolumab for unselected treatmentrefractory GEA in several Asian countries. Pembrolizumab monotherapy in the single arm KEYNOTE-059 phase II study showed similar survival and response outcomes to ATTRACTION-2 (3). In contrast to ATTRACTION-2, KEYNOTE-059 comprised pre-dominantly non-Asian patients and pembrolizumab efficacy appeared dependent on the combined PD-L1 score (CPS) $(3,4)$. This led to accelerated approval of pembrolizumab by the US FDA for patients with treatment-refractory GEA that were CPS $\geq 1$. However, majority of GEA do not respond to single agent ICI and response rates are around $10-15 \%(1,3,5)$. This has contributed to the failure to demonstrate superiority of OS when compared to chemotherapy in the first $(6)$, second $(5,7)$ and third line setting (8) despite more durable responses observed with ICI. Furthermore, median progression-free survival (PFS) appeared shorter with ICI in these studies and the OS curves crossed with an excess early mortality with ICI but a higher proportion of survivors at later timepoints $(5,6)$. Given the limitations of ICI monotherapy in GEA, rationale combinations with chemotherapy to overcome these shortcomings have been tested which has been a successful strategy in other tumour types (9-14). In preclinical tumour models, chemotherapy can induce immunogenic cell death which may be potentiated by ICI (15-20). Furthermore, tumours or their subclones which do not respond to immunotherapy may be controlled by the cytotoxic combination partner. This approach was first demonstrated in the Phase II KEYNOTE-059 cohort 2 which showed promising responses and good tolerability when pembrolizumab was combined with cisplatin and capecitabine or 5 -fluorouracil (21).

Exploring a different chemotherapy doublet with pembrolizumab in the study "Safety and efficacy of pembrolizumab in combination with S-1 plus oxaliplatin as a first-line treatment in patients with advanced gastric/ gastroesophageal junction cancer: Cohort 1 data from the KEYNOTE-659 phase IIb study". Kawazoe et al. present the safety and efficacy of pembrolizumab in combination with S-1 plus oxaliplatin (SOX) cohort in this nonrandomised 2 arm study of Japanese patients with treatment naïve advanced GEA that were HER-2 negative and had tumour CPS $\geq 1$. Patients received pembrolizumab at $200 \mathrm{mg}$ in combination with standard dosing of S-1 $40 \mathrm{mg} / \mathrm{m}^{2}$ and Oxaliplatin $130 \mathrm{mg} / \mathrm{m}^{2}$ (SOX) in a 3-weekly cycle. The primary endpoint was objective response rate (ORR) with other secondary efficacy measures which were descriptive and without a formal hypothesis testing. The regimen was well tolerated with treatment -related adverse 
events similar to that reported with recent trials with S-1 plus oxaliplatin (22-24) suggesting that pembrolizumab did not significantly increase SOX-related toxicity. The frequency of severe immune-related toxicity was also similar to that reported with pembrolizumab monotherapy. However, the relative dose intensity of oxaliplatin was only $59.8 \%$ which is lower than reported (22), this may be partly be explained by the higher dose of oxaliplatin used in this study. A high ORR of $72 \%$ was seen which exceeds that previously observed with SOX $(22,24)$, these responses were also durable with a 6-month PFS of $67 \%$. Due to the short median follow up, the median duration of response and OS was not reached. Interestingly, $57.4 \%$ of subjects had a tumour CPS $\geq 10$ as compared to $27-39 \%$ reported in other studies $(6,7)$. However, tumour biomarkers such as mismatch repair (MMR) status or Epstein-Barr virus (EBV) were not tested. Both of which are associated with higher CPS and response to pembrolizumab (25). Higher CPS has also been correlated with greater responsiveness to pembrolizumab monotherapy (25). Nonetheless, in the exploratory subgroup analysis by Kawazoe et al. the ORR of the chemo combination with pembrolizumab was not higher in patients with CPS $\geq 10$ compared with CPS $\geq 1$ but $<10$, replicating findings from the KEYNOTE-062 (KN-062) study (6). This suggests that the enrichment for higher PD-L1 CPS tumours alone may not explain the high ORR seen in this study and also higher CPS may not be predictive for ORR in ICI-chemotherapy combinations. Furthermore, similarly high ORR and durable responses have also been observed in the Phase II ATTRACTION-4 study which evaluated nivolumab in combination with oxaliplatin and capecitabine or S-1 (26).

Given the remarkable efficacy of ICI combined with chemotherapy as demonstrated in these Phase II studies, can we recommend this in the first line setting? The recent presented Phase III data of the KN-062, Checkmate 649 (CM-649) (27) and ATTRACTION-4 (ATT-4) (28) studies shed some light on this issue. In KN-062, the combination of pembrolizumab and cisplatin and capecitabine or 5 -fluorouracil (5-FU) showed numerically higher median OS (CPS $\geq 1$ HR 0.85 ; CPS $\geq 10$ HR 0.8 ) compared with chemotherapy alone, but did not attain statistical significance (6). ORR also appeared higher with the ICI combination arm (CPS $\geq 1: 48.6 \%$; CPS $\geq 10$ : $52.5 \%$ ) compared with chemotherapy (CPS $\geq 1: 37.2 \%$; $\mathrm{CPS} \geq 10: 37.8 \%)$. In contrast, the interim analysis of CM649 demonstrated that the combination of nivolumab with oxaliplatin and 5-FU or capecitabine significantly improved ORR, PFS and OS in patients CPS $\geq 5$ GEA tumours. Whereas, in the ATT-4 study, the combination of nivolumab with oxaliplatin and capecitabine or TS-1 significantly improved ORR, PFS but not OS compared with chemotherapy alone. Various factors may contribute to these discordant results. Firstly, KN-062 recruited patients with CPS $\geq 1$ GEA while ATT-4 did not select for PDL1 expression but CM-649 was enriched for patients with CPS $\geq 5$ GEA ( $60 \%$ of patients). Secondly, fewer patients in CM-649 went on to receive subsequent ICI. Thirdly, both KN-062 and CM-649 recruited both Western and Asian patients while ATT-4 was conducted in East Asian countries only. It has been observed that there are differences in the immunoprofile of Asian and non-Asian gastric cancers (29) but the data regarding relative benefit of ICI monotherapy in Asians compared with non-Asians is conflicting $(5,6)$. ATT-4 also had the highest proportion of patients receiving subsequent lines of therapy which may have contributed to the lack of OS benefit despite a significant improvement in PFS. Finally, both CM-649 and ATT-4 used oxaliplatin while $\mathrm{KN}-062$ employed cisplatin in the chemotherapy doublet. However, the importance of the choice of platinum is unclear as the KEYNOTE-590 (KN-590) study in esophageal cancers showed PFS and OS benefit for combining pembrolizumab, cisplatin and 5-fluorouracil compared to chemotherapy alone in the adenocarcinoma subgroup particularly in tumours that were CPS $\geq 10$ (30). Taken together the results of these four Phase III studies indicate that patients with advanced GEA with CPS $\geq 5$ benefit from the combination of ICI with doublet chemotherapy in the first line setting. CPS appears to be an important selection biomarker for ICI monotherapy as well. In KEYNOTE-062 the median OS with pembrolizumab in patients with CPS $\geq 10$ tumours appeared longer than the pembrolizumab and chemotherapy combination arm. However, the small sample size and lack of formal statistical testing does not allow conclusion that chemotherapy caused a negative interaction with pembrolizumab efficacy. In the same study, median OS was also noninferior with pembrolizumab compared to chemotherapy in patients with CPS $\geq 1$ tumours. Therefore, CPS may help select some patients in whom pembrolizumab alone in the first line setting may be appropriate, such as elderly patients who may not tolerate doublet chemotherapy. In addition, in the second line setting, subgroup analysis of the KEYNOTE-061 study also demonstrated a trend to better OS and increased and more durable responses with pembrolizumab compared with second line weekly 
paclitaxel in patients with CPS $\geq 5$ and CPS $\geq 10$ GEA (7).

However, the best predictive biomarker for ICI in GEA to date is MMR deficiency. The high and extremely durable responses with single agent pembrolizumab in multiple deficient MMR (dMMR) tumour types in treatmentrefractory disease $(31,32)$ led to its first tumour agnostic approval by the US FDA. In the KN-061, 062 and CM-649 studies, patients with dMMR tumours receiving ICI had greater ORR, PFS, OS and duration of response compared to the chemotherapy alone arm $(5,33)$. Furthermore, the OS with pembrolizumab alone was similar to pembrolizumab combined with chemotherapy in KEYNOTE-062 with less toxicity, suggesting that pembrolizumab monotherapy maybe the preferred choice for patients with dMMR advanced GEA in the first line setting. The recent positive results of the KEYNOTE-177 study in dMMR colorectal cancer (34) lends further support to advance ICI from treatment-refractory disease to the front-line setting of dMMR tumours. Though majority of dMMR tumours have a high tumour mutational burden (TMB), non-dMMR GEA with high TMB also demonstrate high ORR to ICI (35) and pembrolizumab has been approved by the US FDA for high TMB treatment-refractory tumours. EBV-positive GEA are also thought to be highly immunogenic and high response rates to ICI have been observed in small studies $(25,36)$, but currently there is inadequate data to support its use as a selection biomarker in first line therapy. One promising new therapeutic approach has been the combination of ICI with HER2-targeted antibodies $(37,38)$ leveraging on synergy between antibody-dependent cellmediated cytotoxicity and ICI $(37,38)$. High ORR of $87 \%$ were observed with the combination of trastuzumab, pembrolizumab and capecitabine and oxaliplatin in first line treatment of HER2-positive GEA in a Phase II study (38). This multi-modality treatment is currently being evaluated in the first line Phase III KEYNOTE-811 and Phase II/III MAHOGANY studies.

Subject to regulatory approval, the combination of nivolumab and oxaliplatin based doublet chemotherapy may become a new standard for advanced GEA with CPS $\geq 5$ while the combination of pembrolizumab in combination with cisplatin-based doublet chemotherapy may become a new standard for esophageal adenocarcinomas with CPS $\geq 10$ in the first line setting. Whereas, for other GEA, the use of ICI is as monotherapy in the treatment-refractory setting. However, in patients with $\mathrm{dMMR}$ or high CPS tumours, treatment with ICI monotherapy may be a reasonable option in earlier settings. For HER-2 positive
GEA, the ongoing KEYNOTE-811 and MAHOGANY trials will determine if ICI also adds benefit when combined with HER-2 targeted therapy and chemotherapy.

\section{Acknowledgments}

Funding: None.

\section{Footnote}

Provenance and Peer Review: This article was commissioned by the editorial office, Annals of Translational Medicine. The article did not undergo external peer review.

Conflicts of Interest: Both authors has completed the ICMJE uniform disclosure form (available at http://dx.doi. org/10.21037/atm-20-4699). SPC reports personal fees from Eisai, Roche, BMS, Bayer, outside the submitted work. MCHN reports personal fees and other from Bristol Myers Squibbs, from null, outside the submitted work. The authors have no other conflicts of interest to declare.

Ethical Statement: The authors are accountable for all aspects of the work in ensuring that questions related to the accuracy or integrity of any part of the work are appropriately investigated and resolved.

Open Access Statement: This is an Open Access article distributed in accordance with the Creative Commons Attribution-NonCommercial-NoDerivs 4.0 International License (CC BY-NC-ND 4.0), which permits the noncommercial replication and distribution of the article with the strict proviso that no changes or edits are made and the original work is properly cited (including links to both the formal publication through the relevant DOI and the license). See: https://creativecommons.org/licenses/by-nc-nd/4.0/.

\section{References}

1. Kang YK, Boku N, Satoh T, et al. Nivolumab in patients with advanced gastric or gastro-oesophageal junction cancer refractory to, or intolerant of, at least two previous chemotherapy regimens (ONO-4538-12, ATTRACTION-2): a randomised, double-blind, placebocontrolled, phase 3 trial. Lancet 2017;390:2461-71.

2. Chen LT, Satoh T, Ryu MH, et al. A phase 3 study of nivolumab in previously treated advanced gastric or gastroesophageal junction cancer (ATTRACTION-2): 
2-year update data. Gastric Cancer 2020;23:510-9.

3. Fuchs CS, Doi T, Jang RW, et al. Safety and Efficacy of Pembrolizumab Monotherapy in Patients With Previously Treated Advanced Gastric and Gastroesophageal Junction Cancer: Phase 2 Clinical KEYNOTE-059 Trial. JAMA Oncol 2018;4:e180013.

4. Kulangara K, Zhang N, Corigliano E, et al. Clinical Utility of the Combined Positive Score for Programmed Death Ligand-1 Expression and the Approval of Pembrolizumab for Treatment of Gastric Cancer. Arch Pathol Lab Med 2019;143:330-7.

5. Shitara K, Ozguroglu M, Bang YJ, et al. Pembrolizumab versus paclitaxel for previously treated, advanced gastric or gastro-oesophageal junction cancer (KEYNOTE-061): a randomised, open-label, controlled, phase 3 trial. Lancet 2018;392:123-33.

6. Tabernero J, Cutsem EV, Bang YJ, et al. Pembrolizumab with or without chemotherapy versus chemotherapy for advanced gastric or gastroesophageal junction (G/GEJ) adenocarcinoma: The phase III KEYNOTE-062 study. J Clin Oncol 2019;37:LBA4007.

7. Fuchs CS, Özgüroğlu M, Bang YJ, et al. Pembrolizumab versus paclitaxel for previously treated patients with PDL1-positive advanced gastric or gastroesophageal junction cancer (GC): Update from the phase III KEYNOTE-061 trial. J Clin Oncol 2020;38:4503.

8. Bang YJ, Ruiz EY, Van Cutsem E, et al. Phase III, randomised trial of avelumab versus physician's choice of chemotherapy as third-line treatment of patients with advanced gastric or gastro-oesophageal junction cancer: primary analysis of JAVELIN Gastric 300. Ann Oncol 2018;29:2052-60.

9. Burtness B, Harrington KJ, Greil R, et al. Pembrolizumab alone or with chemotherapy versus cetuximab with chemotherapy for recurrent or metastatic squamous cell carcinoma of the head and neck (KEYNOTE-048): a randomised, open-label, phase 3 study. Lancet 2019;394:1915-28.

10. Gandhi L, Garassino MC. Pembrolizumab plus Chemotherapy in Lung Cancer. N Engl J Med 2018;379:e18.

11. Horn L, Mansfield AS, Szczesna A, et al. First-Line Atezolizumab plus Chemotherapy in Extensive-Stage Small-Cell Lung Cancer. N Engl J Med 2018;379:2220-9.

12. Paz-Ares L, Luft A, Vicente D, et al. Pembrolizumab plus Chemotherapy for Squamous Non-Small-Cell Lung Cancer. N Engl J Med 2018;379:2040-51.

13. Schmid P, Adams S, Rugo HS, et al. Atezolizumab and
Nab-Paclitaxel in Advanced Triple-Negative Breast Cancer. N Engl J Med 2018;379:2108-21.

14. Socinski MA, Jotte RM, Cappuzzo F, et al. Atezolizumab for First-Line Treatment of Metastatic Nonsquamous NSCLC. N Engl J Med 2018;378:2288-301.

15. Pfirschke C, Engblom C, Rickelt S, et al. Immunogenic Chemotherapy Sensitizes Tumors to Checkpoint Blockade Therapy. Immunity 2016;44:343-54.

16. Gotwals P, Cameron S, Cipolletta D, et al. Prospects for combining targeted and conventional cancer therapy with immunotherapy. Nat Rev Cancer 2017;17:286-301.

17. Apetoh L, Ghiringhelli F, Tesniere A, et al. Toll-like receptor 4-dependent contribution of the immune system to anticancer chemotherapy and radiotherapy. Nat Med 2007;13:1050-9.

18. Hato SV, Khong A, de Vries IJ, et al. Molecular pathways: the immunogenic effects of platinum-based chemotherapeutics. Clin Cancer Res 2014;20:2831-7.

19. Obeid M, Panaretakis T, Tesniere A, et al. Leveraging the immune system during chemotherapy: moving calreticulin to the cell surface converts apoptotic death from "silent" to immunogenic. Cancer Res 2007;67:7941-4.

20. Zitvogel L, Apetoh L, Ghiringhelli F, et al. Immunological aspects of cancer chemotherapy. Nat Rev Immunol 2008;8:59-73.

21. Bang YJ, Kang YK, Catenacci DV, et al. Pembrolizumab alone or in combination with chemotherapy as firstline therapy for patients with advanced gastric or gastroesophageal junction adenocarcinoma: results from the phase II nonrandomized KEYNOTE-059 study. Gastric Cancer 2019;22:828-37.

22. Yamada Y, Higuchi K, Nishikawa K, et al. Phase III study comparing oxaliplatin plus S-1 with cisplatin plus S-1 in chemotherapy-naive patients with advanced gastric cancer. Ann Oncol 2015;26:141-8.

23. Ryu MH, Park YI, Chung IJ, et al. Phase III trial of s-1 plus oxaliplatin (SOX) vs s-1 plus cisplatin (SP) combination chemotherapy for first-line treatment of advanced gastric cancer (AGC): SOPP study. J Clin Oncol 2016;34:4015.

24. Kim GM, Jeung HC, Rha SY, et al. A randomized phase II trial of S-1-oxaliplatin versus capecitabine-oxaliplatin in advanced gastric cancer. Eur J Cancer 2012;48:518-26.

25. Kim ST, Cristescu R, Bass AJ, et al. Comprehensive molecular characterization of clinical responses to PD-1 inhibition in metastatic gastric cancer. Nat Med 2018;24:1449-58.

26. Boku N, Ryu MH, Kato K, et al. Safety and efficacy 
of nivolumab in combination with $\mathrm{S}-1$ /capecitabine plus oxaliplatin in patients with previously untreated, unresectable, advanced, or recurrent gastric/ gastroesophageal junction cancer: interim results of a randomized, phase II trial (ATTRACTION-4). Ann Oncol 2019;30:250-8.

27. Moehler M, Shitara K, Garrido M, et al. LBA6_PR Nivolumab (nivo) plus chemotherapy (chemo) versus chemo as first-line (1L) treatment for advanced gastric cancer/gastroesophageal junction cancer (GC/GEJC)/ esophageal adenocarcinoma (EAC): First results of the CheckMate 649 study. Ann Oncol 2020;31:S1191.

28. Boku N, Ryu MH, Oh DY, et al. LBA7_PR Nivolumab plus chemotherapy versus chemotherapy alone in patients with previously untreated advanced or recurrent gastric/gastroesophageal junction (G/GEJ) cancer: ATTRACTION-4 (ONO-4538-37) study. Ann Oncol 2020;31:S1192.

29. Lin SJ, Gagnon-Bartsch JA, Tan IB, et al. Signatures of tumour immunity distinguish Asian and non-Asian gastric adenocarcinomas. Gut 2015;64:1721-31.

30. Kato K, Shah MA, Enzinger PC, et al. 785TiP - Phase III KEYNOTE-590 study of chemotherapy + pembrolizumab versus chemotherapy + placebo as first-line therapy for patients (Pts) with advanced esophageal or esophagogastric junction (E/EGJ) cancer. Ann Oncol 2018;29:viii268-9.

31. Marabelle A, Le DT, Ascierto PA, et al. Efficacy of Pembrolizumab in Patients With Noncolorectal High Microsatellite Instability/Mismatch Repair-Deficient Cancer: Results From the Phase II KEYNOTE-158 Study. J Clin Oncol 2020;38:1-10.

32. Le DT, Durham JN, Smith KN, et al. Mismatch repair

Cite this article as: $\mathrm{Ng} \mathrm{MCH}$, Choo SP. Combination of immunotherapy with chemotherapy in first line treatment of metastatic gastric cancer? Too much, too little or just right? Ann Transl Med 2020;8(24):1692. doi: 10.21037/atm-20-4699 deficiency predicts response of solid tumors to PD-1 blockade. Science 2017;357:409-13.

33. Shitara K, Van Cutsem E, Bang YJ, et al. LBA44 Pembrolizumab with or without chemotherapy vs chemotherapy in patients with advanced G/GEJ cancer (GC) including outcomes according to Microsatellite Instability-High (MSI-H) status in KEYNOTE-062. Ann Oncol 2019;30:v878-9.

34. Andre T, Shiu KK, Kim TW, et al. Pembrolizumab versus chemotherapy for microsatellite instability-high/mismatch repair deficient metastatic colorectal cancer: The phase 3 KEYNOTE-177 Study. J Clin Oncol 2020;38:LBA4.

35. Wyrwicz LS, Lee KW, Van Cutsem E, et al. 1442P Association of TMB using the Foundation Medicine Companion Diagnostic (F1CDx) with efficacy of first-line pembrolizumab (pembro) or pembro plus chemotherapy (pembro + chemo) versus chemo in patients with gastric cancer (gc) from KEYNOTE-062. Ann Oncol 2020;31:S907-8.

36. Cancer Genome Atlas Research Network. Comprehensive molecular characterization of gastric adenocarcinoma. Nature 2014;513:202-9.

37. Catenacci DVT, Lim KH, Uronis HE, et al. Antitumor activity of margetuximab $(\mathrm{M})$ plus pembrolizumab $(\mathrm{P})$ in patients (pts) with advanced HER2+ $(\mathrm{IHC} 3+)$ gastric carcinoma (GC). J Clin Oncol 2019;37:65.

38. Janjigian YY, Chou JF, Simmons M, et al. First-line pembrolizumab $(\mathrm{P})$, trastuzumab $(\mathrm{T})$, capecitabine (C) and oxaliplatin (O) in HER2-positive metastatic esophagogastric adenocarcinoma (mEGA). J Clin Oncol 2019;37:62. 